ISSN 1112-9867

\title{
STUDY OF IONIZATION RATES OF HELIUM MAXELLIAN PLASMA
}

\author{
S. Dilmi ${ }^{*}$, E. H. Guedda and A. M. Boumali \\ VTRS et Institut des Sciences et technologie, Centre universitaire d'El-Oued., \\ BP.789, El-Oued 39 000, ALGERIE
}

Received: 06 February 2011 / Accepted: 05 June 2010 / Published online: 30 June 2011

\begin{abstract}
We have contributed to the study and calculation of ionization rate coefficients from the cross sections of neutral helium. Numerical methods and FAC code are used in this work. Our calculations are comparable to published results.
\end{abstract}

\section{INTRODUCTION}

L'hélium neutre est l'élément le plus fréquemment utilisé pour la spectroscopie des plasmas d'astrophysique et de laboratoire. Plusieurs problèmes liés à l'étude des plasmas de laboratoire, d'astrophysique et de fusion thermonucléaire contrôlée nécessitent la connaissance au préalable de la structure atomique comme les énergies et longueurs d'onde des différents niveaux et les sections efficaces et les coefficients de taux des processus élémentaires dans les plasmas. Cependant, le calcul direct de ces données devient impossible pour les atomes à plusieurs électrons et aussi pour les grands nombres quantiques. Dans ce cas on fait recours aux programmes et codes de calcul. Malgré le nombre important des codes existant pour le traitement des données atomiques, ils sont tous en compétition pour donner des résultats proches de l'expérimental.

Author Correspondence, e-mail: sam1dilmi@yahoo.fr

ICID: 1020769 
Le code FAC (Flexible Atomic Code) [2] a été choisi pour calculer les sections efficaces d'ionisation de l'helium neutre. Les taux d'ionisation de l'hélium seront générés par un calcul numérique.

\section{ASPECT THEORIQUE}

Dans le processus d'ionisation par l'électron d'impact, un atome ou ion peut heurter_un électron, il s'ionise en cédant aussi un électron :

$$
(\mathrm{Z}, \mathrm{N})+\mathrm{e} \Leftrightarrow(\mathrm{Z}, \mathrm{N}-1)+\mathrm{e}+\mathrm{e}
$$

où $(\mathrm{Z}, \mathrm{N})$ représente un ion de charge $\mathrm{Z}$ possédant $\mathrm{N}$ électrons.

Le phénomène inverse de l'ionisation est la recombinaison à trois corps. Les sections efficaces et les coefficients de taux sont obtenus par le principe d'équilibre détaillé à partir des sections efficaces et les coefficients de taux des processus d'ionisation correspondants. Les sections efficaces d'ionisation par l'électron d'impact peuvent être calculées par le programme FAC [2] utilisant l'approximation relativiste des ondes déformées avec une méthode d'interpolation-factorisation. Ceci permet d'inclure la recombinaison entre configurations [3].

Le coefficient de taux d'ionisation $\tau$ est donné en fonction de la section efficace d'ionisation $\sigma(E)$ par l'équation :

$$
\tau=\int v \sigma(E) f(E) d E \quad\left(\mathrm{~cm}^{3} \mathrm{~s}^{-1}\right)
$$

Où;

$$
v=\sqrt{\frac{2 E}{M}} \quad ; \quad f(E) d E=\frac{2 \sqrt{E} e^{-\frac{E}{T}}}{T^{3 / 2} \sqrt{\pi}} d E
$$

où $v$ est la vitesse des particules de masse $\mathrm{M}$; $\mathrm{T}$ est la température en $(\mathrm{eV})$; $\mathrm{E}$ est l'énergie 'Threshold' d'ionisation de l'hélium; et $f(E)$ est la distribution maxwellienne pour l'énergie.

\section{CALCUL DES COEFFICIENTS DE TAUX D'IONISATION DE L'HELIUM NEUTRE}

\section{3-1- Méthode de calcul}

Les sections efficaces d'ionisation de l'hélium sont obtenues par le code FAC dans la gamme énergétique $\left(10-10^{5} \mathrm{eV}\right)$. Le tableau 1 présente quelques valeurs des ces sections efficaces d'ionisation. Les coefficients de taux d'ionisation seront calculés suivant l'équation (2). Le calcul numérique de l'intégral présenté par l'équation (2) est basé sur la méthode de trapèze. 
Tableau 1. Sections efficaces d'ionisation de l'hélium

\begin{tabular}{|l|l|}
\hline Energie $(\mathrm{eV})$ & Section efficace $\left[10^{-20}\right]\left(\mathrm{cm}^{2}\right)$ \\
\hline 56 & $5.65810^{3}$ \\
146 & $4.54610^{3}$ \\
546 & $2.04810^{3}$ \\
$5210^{3}$ & 927.21 \\
$1610^{4}$ & 61.107 \\
& \\
\hline
\end{tabular}

\section{RESULTATS ET DISCUTIONS}

Les résultats de calcul des taux d'ionisation de l'hélium obtenus seront reportés sur la figure 1. Ces résultats seront comparés à ceux publiés par Kato et al. [4]. En général, une bonne concordance a été remarquée sur les deux courbes de la figure 1. Cependant le tableau 2 montre les erreurs relatives entre les taux de Kato et al. ( $\boldsymbol{\tau}_{\text {Kato }}$ ) et ceux du présent travail $(\boldsymbol{\tau})$. Les erreurs ne dépassent pas $40 \%$ dans la gamme énergétique $5 \mathrm{eV}$ $10 \mathrm{eV}$. Elles sont aussi trop faibles de l'ordre de $0.1 \%$ dans l'intervalle [200eV, $1000 \mathrm{eV}$. Alors que ces erreurs sont considérables dans la gamme $12 \mathrm{eV}-80 \mathrm{eV}$.

Tableau 2. Les erreurs relatives des taux d'ionisation.

\begin{tabular}{|l|l|l|l|}
\hline $\mathrm{T}(\mathrm{eV})$ & $\tau$ & $\tau_{\text {Kato }}$ & $\tau / \tau$ \\
\hline 7.0409 & $1.353910^{-10}$ & $2.418410^{-10}$ & 0.4400 \\
10.1369 & $7.7160110^{-10}$ & $1.006510^{-9}$ & 0.2333 \\
\hline 12.0667 & $1.887210^{-9}$ & $1.250810^{-9}$ & 0.5087 \\
50.2527 & $2.064010^{-8}$ & $1.273210^{-8}$ & 0.6211 \\
100.9661 & $2.692410^{-8}$ & $2.114710^{-8}$ & 0.2731 \\
\hline 205.9784 & $2.758510^{-8}$ & $2.445910^{-8}$ & 0.1278 \\
326.2352 & $2.758510^{-8}$ & $2.758510^{-8}$ & 0.0000 \\
1000 & $2.330110^{-8}$ & $2.330110^{-8}$ & 0.0000 \\
\hline
\end{tabular}




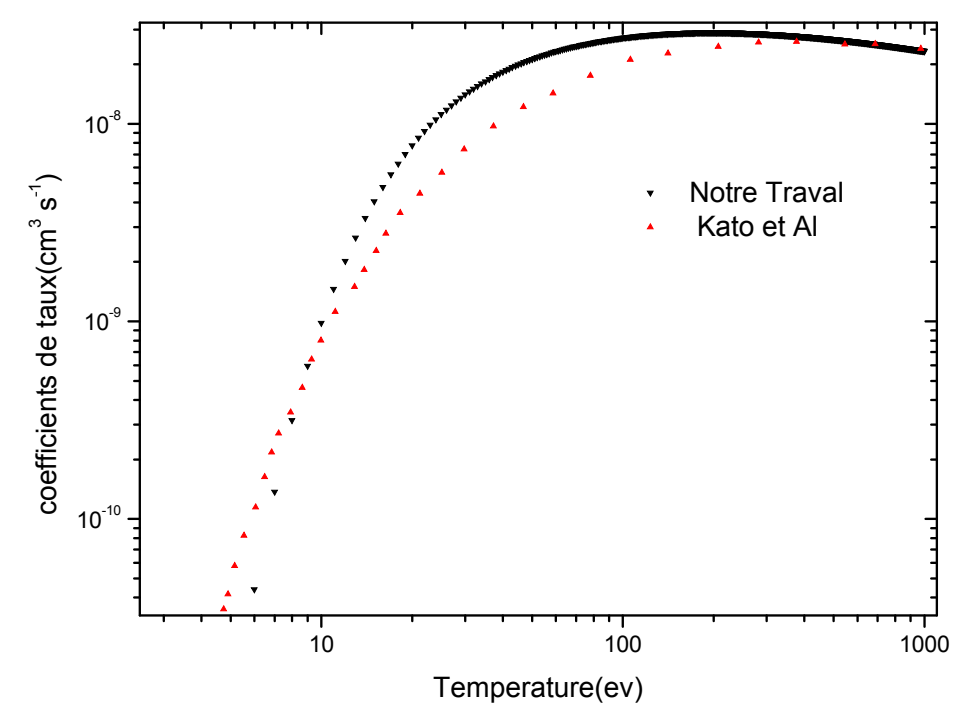

Fig.1. Coefficients de taux d'ionisations pour l'He

\section{CONCLUSION}

Les coefficients de taux d'ionisation de l'hélium ont été calculés à partir des sections efficaces. Ces dernières ont été obtenues par le code FAC. Les résultats obtenus sont, généralement, en bonne concordance avec les résultats publiés.

\section{BIBLIOGRAPHIE}

[1] Guedda E.H. «Contribution à des structures atomiques et processus élémentaires dans les plasmas: Applications aux hydrogénoides et aux héliumoides »; thèse de doctorat, Université Badji Mokhtar Annaba, (2006).

[2] M. F. Gu, FAC 1.0.7, http://kipac-tree.stanford.edu/fac/

[3] M. Arnaud and R. Rothenflug, Astron, Astrophys. Supp. Ser. 60 (1985) 425.

[4] T. Kato et al. NIFS-Data-Series, 14 (1991). 


\section{ETUDE DES TAUX D'IONISATION D'UN PLASMA MAXWELLIEN D'HELIUM}

\section{RESUME}

Nous avons contribué à l'étude et au calcul des coefficients des taux d'ionisation de l'hélium neutre à partir des sections efficaces. Le travail présenté est basé sur des méthodes numériques et le code FAC. Nos calculs sont comparables aux résultats publiés.

Mots clés: He neutre, Sections efficaces d'ionisation, coefficients des taux d'ionisation, FAC.

How to cite this article Dilmi S, Guedda E H, Boumali A M. Study of ionization rates of helium maxellian plasma. J Fundam Appl Sci. 2011, 3(1), 14-18. 